\title{
Ophthalmological problems in pregnancy - a review
}

\author{
Anna Rzeszotarska®, Justyna Szczapa-Jagustyn®i, Jaroslaw Kociecki® \\ Ophthalmology Institute and Clinic of Karol Marcinkowski University of Medical Sciences in Poznan, Poland
}

\begin{abstract}
Pregnancy is associated with numerous changes affecting all organs. Ophthalmological changes in pregnant women are most often physiological and resolve spontaneously after delivery. However, the possibility of progression of previously diagnosed ophthalmic diseases or the occurrence of ophthalmological complications in the course of diseases characteristic for pregnancy should always be considered.
\end{abstract}

Key words: pregnancy; eye; glaucoma; diabetic retinopathy; myopia; preeclampsia; eclampsia; Sheehan's syndrome; papilloedema; central serous retinopathy

Ginekologia Polska 2020; 91, 8: 473-477

\section{INTRODUCTION}

Ophthalmological changes during pregnancy are usually physiological, temporary and do not require any treatment. However, in some women with previously diagnosed glaucoma or diabetic retinopathy there is a great risk of illness progression. Also, some medical conditions typical for pregnancy, such as preeclampsia, eclampsia and Sheehan's syndrome, may manifest with ophthalmological symptoms.

The main aim of this review is to present possible pathological changes ophthalmological diseases progression that may occur throughout pregnancy. Moreover, we would like to discuss possible treatment methods of some disorders and the ophthalmological indications for caesarean section.

\section{Glaucoma and pregnancy}

Glaucoma is neuropathy of the optic nerve characterized by typical changes in the visual field and optic nerve head that usually occur in people over 40 years of age but may present in younger patients. Glaucoma treatment is based on pharmacotherapy or surgical techniques, including laser treatment. The only modifiable risk factor in glaucoma patients is the intraocular pressure (IOP). Persisting high values of IOP lead to glaucoma progression. In many cases pharmacotherapy is adequate to maintain the target IOP. During pregnancy the IOP decreases but many women with diagnosed glaucoma still require further treatment.

\section{Glaucoma pharmacotherapy}

Prostaglandins, beta blockers, alpha-2 agonists, carbonic anhydrase inhibitors, miotics and osmotic agents are used in glaucoma pharmacotherapy. Most of them are applied topically to the conjunctival sac. To obtain maximum local treatment and minimize the general adverse effects, proper application is essential. Inserting only one drop of antiglaucoma drug, closing the eye for 3-5 minutes and compressing the lacrimal puncta all help to reduce systemic absorption.

Brimonidine is one of the alpha- 2 agonists classified as category B by the Food and Drug Administration (FDA). This drug may be used during pregnancy but therapy with brimonidine should be ceased before labour because it penetrates through the blood-brain barrier, which can cause apnoea in the newborn [1].

Among the beta blockers used in glaucoma pharmacotherapy there are several that are non-selective, such as timolol, carteolol, levobunolol, metipranolol and beta-1-selective betaxolol, all of which are classified as category $C$ by the FDA. There are limited reports about the impact of locally administered beta blockers on the foetus, however extreme caution must be exercised, especially in the first trimester, due to their teratogenic effect. Moreover, if given systemically during the second or third trimester, beta blockers may lead to intrauterine growth restriction (IUGR), as well as dysfunction of the newborn's cardiovascular and nervous system. It is assumed that even small doses of timolol used by pregnant women lead to permanent changes in the heart's electrical conduction system [2]. Furthermore, 
breathing disorders were observed in newborns whose mothers used timolol during pregnancy [3].

Prostaglandin analogues (travoprost, latanoprost, bimatoprost, tafluprost) are very effective hypotensive drugs commonly used in glaucoma therapy, although their usage is not recommended in pregnant women. An increased number of miscarriages were observed, compared to a control group, when the prostaglandins were used topically in animal research [4]. Prostaglandin analogues may increase uterine tonus and stimulate uterine contractions, leading to miscarriage or premature labour.

Carbonic anhydrase inhibitors can be used both topically and systemically (orally or intravenously). Brinzolamide and acetazolamide are classified as category $C$ by the FDA. Animal testing with brinzolamide revealed the presence of additional cranial bones in rabbit foetuses, however the dose was higher than that used in antiglaucoma eye drops [5]. Acetazolamide can be used topically, orally or intravenously. During animal testing with acetazolamide, front extremity defects in rat foetuses were observed after high doses [6]. Warsham et al. [7] published the case report of a newborn with sacrococcygeal teratoma whose mother used acetazolamide throughout her pregnancy. There is also a risk of metabolic disorders in newborns if the mother used acetazolamide whilst pregnant [8].

Pilocarpine is quite rarely used in glaucoma treatment, mainly for acute angle closure. Like other parasympathomimetic drugs, it is classified as category $C$ by the FDA. In animal testing, pilocarpine's teratogenic action has been proved [9].

In order to achieve fast reduction of the IOP, osmotic drugs such as mannitol or $85 \%$ glycerol solution are commonly used. Both drugs are category $C$ and should be used during pregnancy only if the benefits overweigh the risks.

\section{Surgical treatment in glaucoma patients}

Surgical treatment for glaucoma includes both laser and surgical methods.

Laser treatments such as selective laser trabeculoplasty (SLT) or YAG-laser iridotomy are possible throughout pregnancy. SLT allows the number of antiglaucoma drugs to be reduced or, in some cases, eliminated completely. Iridotomy is a well-known procedure performed in patients with a narrow or closed iridocorneal angle. Laser treatment seems to be a safe method for managing glaucoma but both SLT and iridotomy require perioperative medication with topical drugs, some of which are category $C$.

If progression in visual field changes is observed despite maximal pharmacological treatment with topical drugs, or if drops are not well tolerated and cause local or systemic side effects, then surgery should be considered. Many of the filtration surgeries, such as trabeculectomy, phacotra- beculectomy and deep sclerectomy, require the use of antimetabolites (mitomycin C or 5-fluorouracil) during the procedure to achieve a better effect and reduce the risk of scarring. Unfortunately, antimetabolites have a teratogenic and mutagenic effect on the foetus $[10,11]$. It seems that the best option is to conduct an operation before any planned conception. Other surgical techniques used in advanced glaucoma, such as cyclodestruction procedures (cyclocryoapplication or the much more popular cyclophotocoagulation), should be preceded by periocular anaesthesia with lidocaine or bupivacaine, which may be associated with serious side effects in both the mother and child.

\section{Labour and glaucoma}

One of the surveys conducted on a group of healthy pregnant women revealed a statistically significant increase in IOP during labour [12]. Vaginal delivery in women with a narrow iridocorneal angle is associated with a high risk of acute angle closure [13]. Furthermore, decreased intraocular blood flow caused by postpartum haemorrhage is extremely dangerous. Caesarean section should be considered in patients with advanced glaucoma in order to prevent further ocular complications.

What are the indications for caesarean section in patients with glaucoma? According to the Ophthalmologic and Obstetrical Consensus on Indications for Delivery by Caesarean Section due to Changes in the Sight Organ, February 2017 [14], the decision is made by an experienced obstetrician based on the opinion of an ophthalmologist after ophthalmological examination. Patients with advanced changes in the visual field caused by glaucoma have a high risk of ophthalmological complications if they give birth vaginally. Each case should be considered individually after appropriate examination and careful analysis of previous medical records to determine the potential degree of disease progression.

\section{Diabetic retinopathy in pregnant women}

Gestational diabetes mellitus (GDM) is defined as the onset or first diagnosis during pregnancy of glucose intolerance. If diagnosed before conception, the diabetes is defined as pregestational diabetes mellitus (PGDM).

GDM does not require monitoring throughout pregnancy. In a survey of 107 pregnant women diagnosed with GDM, none of the patients developed diabetic retinopathy (DR) during pregnancy [15].

However, with PGDM (regardless of type), pregnancy is a significant risk factor for DR progression due to many factors: poor glycaemic control, duration of diabetes, fast normoglycaemia achievement during the first weeks of pregnancy, elevated serum levels of growth factors, co-existing hypertension or preeclampsia and haemodynamic changes 
in blood vessels. Improper glycaemic control by a woman of conceptual age may lead to DR development before pregnancy [16]. Obtaining normoglycaemia decreases the risk of miscarriage or congenital defects but, paradoxically, is associated with DR progression. The duration of diabetes seems to be less relevant compared with the previously discussed risk factors. It has been proved that increased growth factor serum levels such as IGF-1 cause progression of DR despite good glycaemic control [17]. Hypertension is critical in DR progression, especially in pregnancy. In one cohort study of a group of 154 pregnant women diagnosed with both pregestational DR and hypertension, progression of retinal changes was observed in 55\%. On the other hand, in women diagnosed with PGDM but with no hypertension, progression of DR occurred in only 25\% [18]. Furthermore, haemodynamic changes characteristic for pregnancy, such as increased cardiac output, increased plasma volume and reduced peripheral vascular resistance, affect the retinal blood flow. In healthy pregnant women there are mechanisms that maintain a constant blood flow [19] but a hyperdynamic blood circulation in pregnant women with PGDM can cause further endothelial damage [20].

In 2015 the National Institute of Clinical Excellence (NICE) published new guidelines for women with PGDM [21]. According to the NICE guidelines all women with previously diagnosed diabetes should be informed about potential DR progression in the preconception period. Also, women with diabetes who are planning a pregnancy should accomplish good glycaemic control before conception. Moreover, patients should be informed about the risk of DR progression caused by rapidly achieving normoglycaemia. The authors of the NICE 2015 Guidelines advise that the first fundoscopy should be conducted after an earlier application of mydriatics (e.g. tropicamide) during the first visit, optimally in the $10^{\text {th }}$ week of pregnancy, and then also in the 28th week of pregnancy. If during the first ophthalmological examination there are any symptoms indicating DR, then an additional fundoscopy should be conducted between the 16th and 20th week of pregnancy. The presence of features indicating DR are contraindications for rapid glycaemia and glycated haemoglobin (HbA1c) optimization. All women with diagnosed DR or with any retinal changes due to DR during pregnancy should be monitored for six months after labour.

Optimal glycaemic control and proper treatment of previously diagnosed DR before a planned pregnancy should prevent any DR progression that requires treatment. In one retrospective study conducted on 540 pregnant women with diabetes over a 12-year period, only eight patients (1.5\%) experienced changes characteristic of proliferative retinopathy requiring retinal laser therapy [22]. Decisions about laser treatment of the retina in such cases should not be delayed because proliferative DR is associated with a great risk of complications and the need for extensive vitreoretinal surgery.

Diabetic macular oedema (DMO) occurs in a minor number of pregnant women with diabetes and subsides spontaneously after labour [23]. Co-existing nephropathy and hypertension are important risk factors for DMO in GDM. Intravitreal injections of anti-vascular endothelial growth factor (anti-VEGF) - ranibizumab, bevacizumab, aflibercept - are widely used in DMO treatment, although animal tests demonstrate adverse effects on embryo and foetus, therefore they should not be used during pregnancy.

According to the NICE Guidelines 2015, uncomplicated DR is not a direct indication for spontaneous delivery [21]. On the other hand, the authors of the Ophthalmologic and Obstetrical Consensus on Indications for Delivery by Caesarean Section due to Changes in the Sight Organ recommend that ending pregnancy by caesarean section should be considered in the following cases: the presence of recurrent vitreous haemorrhage due to DR; the presence of neovascularization at the disk (NVD) or neovascularization elsewhere (NVE), which probably will not disappear (after treatment or spontaneously) before delivery; and the presence of tractional retinal detachment that develops and progresses throughout pregnancy [14].

\section{Central serous retinopathy}

Central serous retinopathy (CSR) is an idiopathic disease characterized by retinal detachment in the macular region secondary to focal defect of the retinal pigment epithelium. CSR is typical for males aged $20-50$ years but it can also be observed in females. The risk factors of CSR are stress, steroid therapy, Cushing syndrome, hypertension and gestation. Presented symptoms such as blurry vision, central scotoma, micropsia and metamorphopsia are usually unilateral.

In one prospective study it has been estimated that CSR affects $0.44 \%$ of pregnant women [24]. The best diagnostic method to confirm CSR is fluorescein angiography, however optical coherence tomography (OCT) seems to be a safer option.

CSR is usually self-limiting, with all symptoms generally resolving within three months. However, if CSR symptoms do not resolve there are a few therapeutic methods that might be considered, such as laser therapy or pharmacological treatment (using spironolactone, eplerenone, finasteride, carbonic anhydrase inhibitor or acetylsalicylic acid) [25]. When choosing a specific therapy for CSR in pregnant women, the risk of foetal defects must be considered.

\section{Myopia}

Myopia is a common refractive error caused by both genetic and environmental factors and is classified as low (less than -2 dioptre), moderate ( -2 to -6 dioptre) or high 
(more than -6 dioptre). In a survey, Fernandez-Montero et al. [26] found that pregnancy is a risk factor for myopia development or progression. Another survey conducted by Piazzello [27] found that a myopic shift was observed in pregnant women but that it returned to pre-pregnancy values after labour.

Although high myopia is a risk factor for retinal detachment, it is not a contraindication for spontaneous vaginal delivery. Neri et al. [28] examined 50 women with myopia ranging from $-4,5$ to -15 dioptre, with each patient undergoing fundoscopy before and after labour; none of them had a retinal detachment or tear caused by the delivery.

In some patients with degenerative myopia, choroidal neovascularization may develop and caesarean section should be performed [14]; otherwise, even high myopia should not be considered an indication for caesarean section.

\section{Papilloedema during pregnancy}

Papilloedema is always a diagnostic challenge and is defined as optic disc oedema secondary to increased intracranial pressure. Optic disc swelling may be caused by both life-threatening and non-life-threatening conditions and requires complex diagnostics. There are many causes of papilloedema in pregnant women, some of which are related to the pregnancy. For a differential diagnosis, malignant hypertension, mass lesion and obstructive hydrocephalus should be taken into consideration; however, the most common causes of papilloedema are idiopathic intracranial hypertension (IHT) and central venous thrombosis (CVT) [29].

Evaluating the blood pressure and tests for proteinuria should be conducted to exclude preeclampsia. MRI of the brain without contrast is a safe diagnostic procedure after 18 weeks of pregnancy [31] for detecting mass lesions, hydrocephalus or CVT but there are few data confirming the safety of MRI without contrast in the first trimester. Due to exposure to radiation and a teratogenic effect on the foetus, computed tomography (CT) should only be conducted if MRI is inaccessible. Proper preventive methods should be used to avoid radiation to the foetus. Lumbar puncture (LP) is also a possible diagnostic method in pregnant women if any contraindications are found after evaluating the MRI or CT scans; after obtaining cerebrospinal fluid (CSF) several laboratory tests (glucose, cell, protein count, cytology, VDRL, cryptococcal antigen) should be conducted.

Treatment depends on diagnosis of the primary disorder that leads to papilloedema and must be undertaken to avoid loss of optic nerve function. Diuretics, steroids and anticoagulants are used in the treatment of IHT or CVT. Many of these drugs are classified as category $C$, which is why there is always a probable risk of side effects on the foetus [29].
Ocular symptoms of preeclampsia and eclampsia

Blurry vision, photopsia and visual field defects are symptoms that may occur in women with eclampsia or preeclampsia. Fundoscopy reveals retinal haemorrhages, Elschnig spots, macular oedema, cotton wool spots and segmented or generalized blood vessel narrowing. In some cases, papilloedema or serous retinal detachment is also observed [31].

The most crucial for therapy is obtaining an optimal blood pressure level. Macular oedema may be treated with laser therapy, steroids or anti-VEGF intravitreal injections after carefully reviewing the benefits and risks. Serous retinal detachment should spontaneously subside after curing the underlying disease.

\section{Sheehan's syndrome}

An ocular manifestation of Sheehan's syndrome can be diplopia (double vision), sudden vision loss or visual field defects and ophthalmoplegia. Medical history that suggests perinatal haemorrhage requires proper diagnostics regarding assessment of thyroid and adrenal gland function, level of growth hormone and MRI scan results [32]. Ocular symptoms of Sheehan's syndrome may recede after using hydrocortisone [33].

\section{SUMMARY}

Ocular disorders in pregnant women are most often physiological and transient. However, severe eye disorders during pregnancy should always be considered and treated in order to avoid serious complications. In patients with diabetes, hypertension, glaucoma, papilloedema or retinal detachment, constant ophthalmological evaluation is necessary throughout pregnancy.

\section{REFERENCES}

1. Cantor LB, Safyan E, Liu CC, et al. Brimonidine in the treatment of glaucoma and ocular hypertension. Ther Clin Risk Manag. 2006; 2(4): 337-346, doi: 10.2147/tcrm.2006.2.4.337, indexed in Pubmed: 18360646.

2. Wagenvoort $A M$, van Vugt JM, Sobotka $M$, et al. Topical timolol therapy in pregnancy: is it safe for the fetus? Teratology. 1998; 58(6): 258-262, doi: 10.1002/(SICI) 1096-9926(199812)58:6<258::AID-TERA7>3.0.CO;2-B, indexed in Pubmed: 9894675.

3. Olson R, Bromberg BB, Zimmerman T. Apneic Spells Associated with Timolol Therapy in a Neonate. American Journal of Ophthalmology. 1979; 88(1): 120-122, doi: 10.1016/0002-9394(79)90766-9.

4. Sharif NA. Synthetic FP-prostaglandin-induced contraction of rat uterus smooth muscle in vitro. Prostaglandins Leukot Essent Fatty Acids. 2008; 78(3): 199-207, doi: 10.1016/j.plefa.2008.01.005, indexed in Pubmed: 18375109.

5. Alcon Ophthalmics. Azopt Product Monograph. Fort Worth, TX: Alcon Ophthalmics. ; 1998.

6. Weaver TE, Scott WJ. Acetazolamide teratogenesis: interaction of maternal metabolic and respiratory acidosis in the induction of ectrodactyly in C57BL/6J mice. Teratology. 1984; 30(2): 195-202, doi: 10.1002/tera.1420300207, indexed in Pubmed: 6436998.

7. Worsham G. Sacrococcygeal Teratoma in a Neonate. JAMA. 1978; 240(3): 251, doi: 10.1001/jama.1978.03290030069029. 
8. Capino AC, Dannaway DC, Miller JL. Metabolic Acidosis with Ophthalmic Dorzolamide in a Neonate. J Pediatr Pharmacol Ther. 2016; 21(3): 256259, doi: 10.5863/1551-6776-21.3.256, indexed in Pubmed: 27453705.

9. Landauer W. The teratogenic activity of pilocarpine, pilocarpidine and their isomers, with special reference to the importance of steric configuration. Journal of Experimental Zoology. 1956; 132(1): 39-50, doi: 10.1002/jez.1401320104.

10. Kuwagata $\mathrm{M}$, Takashima $\mathrm{H}$, Nagao $\mathrm{T}$. A comparison of the in vivo and in vitro response of rat embryos to 5-fluorouracil. J Vet Med Sci. 1998; 60(1): 93-99, doi: 10.1292/jvms.60.93, indexed in Pubmed: 9492366.

11. Shepard TH, Lemire RJ. Catalog of Teratogenic Agents, 2nd edn. Baltimore, MD: Johns Hopkins University Press, 2004, p. ; 278.

12. Avasthi $P$, Sethi $P$, Mithal S. Effect of pregnancy and labor on intraocular pressure. Int Surg. 1976; 61(2): 82-84, indexed in Pubmed: 1254403.

13. Kearns PP, Dhillon BJ. Angle closure glaucoma precipitated by labour. Acta Ophthalmol (Copenh). 1990; 68(2): 225-226, doi: 10.1111/j.17553768.1990.tb01910.x, indexed in Pubmed: 2356714.

14. Ophthalmologic and obstetric consensus on indications for caesarean section. Warsaw: Polish Ophthalmological Society. ; 2017.

15. Horvat $M$, Maclean $H$, Goldberg $L$, et al. Diabetic retinopathy in pregnancy: a 12-year prospective survey. Br J Ophthalmol. 1980; 64(6): 398-403, doi: 10.1136/bjo.64.6.398, indexed in Pubmed: 7387964.

16. Chew EY, Mills JL, Metzger BE, et al. Metabolic control and progression of retinopathy. The Diabetes in Early Pregnancy Study. National Institute of Child Health and Human Development Diabetes in Early Pregnancy Study. Diabetes Care. 1995; 18(5): 631-637, doi: 10.2337/diacare.18.5.631, indexed in Pubmed: 8586000.

17. Lauszus FF, Klebe JG, BekT, et al. Increased serum IGF-I during pregnancy is associated with progression of diabetic retinopathy. Diabetes. 2003; 52(3): 852-856, doi: 10.2337/diabetes.52.3.852, indexed in Pubmed: 12606530.

18. Rosenn B, Miodovnik M, Kranias G, et al. Progression of diabetic retinopathy in pregnancy: association with hypertension in pregnancy. Am J Obstet Gynecol. 1992; 166(4): 1214-1218, doi: 10.1016/s00029378(11)90608-5, indexed in Pubmed: 1566772.

19. Chen HC, Newsom RS, Patel V, et al. Retinal blood flow changes during pregnancy in women with diabetes. Invest Ophthalmol Vis Sci. 1994; 35(8): 3199-3208, indexed in Pubmed: 8045714.

20. Tooke JE. Microvascular function in human diabetes. A physiological perspective. Diabetes. 1995; 44(7): 721-726, doi: 10.2337/diab.44.7.721, indexed in Pubmed: 7789639.
21. https://www.nice.org.uk/guidance/ng3/resources/diabetes-in-pregnancy-management-from-preconception-to-the-postnatal-period-51038446021.

22. Chan WC, Lim LT, Quinn MJ, et al. Management and outcome of sight-threatening diabetic retinopathy in pregnancy. Eye (Lond). 2004; 18(8):826-832, doi: 10.1038/sj.eye.6701340, indexed in Pubmed: 14976547.

23. Sinclair SH, Nesler C, Foxman B, et al. Macular edema and pregnancy in insulin-dependent diabetes. Am J Ophthalmol. 1984; 97(2): 154-167, doi: 10.1016/s0002-9394(14)76085-4, indexed in Pubmed: 6696026.

24. Said-Ahmed K, Moustafa G, Fawzy M. Incidence and natural course of symptomatic central serous chorioretinopathy in pregnant women in a maternity hospital in Kuwait. Middle East Afr J Ophthalmol. 2012; 19(3): 273-276, doi: 10.4103/0974-9233.97920, indexed in Pubmed: 22837618

25. Abouammoh MA. Advances in the treatment of central serous chorioretinopathy. Saudi J Ophthalmol. 2015; 29(4): 278-286, doi: 10.1016/j. sjopt.2015.01.007, indexed in Pubmed: 26586979.

26. Fernández-Montero $A$, Bes-Rastrollo $M$, Moreno-Montañés J, et al. Effect of pregnancy in myopia progression: the SUN cohort. Eye (Lond). 2017; 31(7): 1085-1092, doi: 10.1038/eye.2017.24, indexed in Pubmed: 28304386.

27. Pizzarello LD. Refractive changes in pregnancy. Graefes Arch Clin Exp Ophthalmol. 2003; 241(6): 484-488, doi: 10.1007/s00417-003-0674-0, indexed in Pubmed: 12736728.

28. Neri A, Grausbord R, Kremer I, et al. The management of labor in high myopic patients. Eur J Obstet Gynecol Reprod Biol. 1985; 19(5): 277-279, doi: 10.1016/0028-2243(85)90041-3, indexed in Pubmed: 4018367.

29. Schiffman JS, Scherokman B, Tang RA, et al. Evaluation and treatment of papilledema in pregnancy. Compr Ophthalmol Update. 2006; 7(4): 187-202, indexed in Pubmed: 17007732.

30. Principles for the protection of patients and volunteers during clinical magnetic resonance diagnostic procedures. Ann NY Acad Sci. 1992; 649:372-375, doi: 10.1111/j.1749-6632.1992.tb49634.x, indexed in Pubmed: 1580515.

31. Abu Samra K. The eye and visual system in the preeclampsia/eclampsia syndrome: What to expect? Saudi J Ophthalmol. 2013; 27(1):51-53, doi: 10.1016/j.sjopt.2012.04.003, indexed in Pubmed: 23964188.

32. Krysiak R, Okopień B. [Sheehan's syndrome--a forgotten disease with 100 years' history]. Przegl Lek. 2015; 72(6): 313-320, indexed in Pubmed: 26817341.

33. Vaphiades MS, Simmons D, Archer RL, et al. Sheehan syndrome: a splinter of the mind. Surv Ophthalmol. 2003; 48(2): 230-233, doi: 10.1016/s00396257(02)00459-9, indexed in Pubmed: 12686307. 